\title{
Plasma deposition of thin carbonfluorine films on aligned carbon nanotube
}

\author{
Peng He \\ Department of Mechanical Engineering, University of Cincinnati, Cincinnati, Ohio 45221 \\ Donglu Shi ${ }^{\mathrm{a})}$ \\ Department of Chemical and Materials Engineering, University of Cincinnati, Cincinnati, Ohio 45221 \\ Jie Lian, L. M. Wang, and Rodney C. Ewing \\ Departments of Geological Sciences and Material Science \& Engineering, University of Michigan, Ann \\ Arbor, Michigan 48109 \\ Wim van Ooij \\ Department of Chemical and Materials Engineering, University of Cincinnati, Cincinnati, Ohio 45221 \\ W. Z. Li \\ Department of Physics, Florida International University, Miami, Florida 33199 \\ Z. F. Ren \\ Department of Physics, Boston College, Boston, Massachusetts 02467
}

(Received 15 July 2004; accepted 16 November 2004; published online 19 January 2005)

\begin{abstract}
The thin film of carbonfluorine was deposited on the surfaces of aligned carbon nanotubes using a plasma polymerization treatment. High-resolution transmission electron microscopy images revealed that a thin film of the polymer layer $(20 \mathrm{~nm})$ was uniformly deposited on the surfaces of the aligned carbon nanotubes. Time-of-flight secondary ion mass spectroscopy and Fourier transform infrared experiments identified the carbonfluorine thin films on the carbon nanotubes. The plasma deposition mechanism is discussed. (C) 2005 American Institute of Physics.
\end{abstract}

[DOI: $10.1063 / 1.1846957]$

The development of surface nanostructure will be one of the key engines that drive our technological society in the 21 st century. This rapidly growing area focuses on tailoring a nanoparticle surface structure for specific and unique properties. ${ }^{1-5}$ One of the important developments in nanostructures is the synthesis of carbon nanotubes. Carbon nanotubes are intrinsically superelastic one-dimensional structures that are chemically inert, and they possess electrochemical, piezoelectric, tunable electronic and high thermal conductivity properties. ${ }^{6}$ These properties make carbon nanotubes the potential successor to carbon fibers in composites, silicon in electronic devices, ionic polymers for actuators, ${ }^{7}$ and a myriad of other technologies for sensors. Unfortunately, the surface of the nanotubes is often not ideal for a particular application. The ability to deposit wellcontrolled coatings on nanotubes would offer a wide range of technological opportunities, based on changes to both the physical and chemical properties of the nanotubes and nanoparticles.

In our previous works, several nanoparticles and carbon nanotubes have been successfully coated with plasma polymerization. ${ }^{8-12}$ In this letter, we present experimental results on the plasma coating of aligned carbon nanotubes. High-resolution transmission electron microscopy (HRTEM), time-of-flight secondary ion mass spectroscopy (TOF-SIMS), and Fourier transform infrared (FTIR) experimental results are presented on the surface thin film deposition and characterization. The deposition mechanisms are also discussed.

In this experiment, the aligned carbon nanotubes were synthesized on silicon substrates by plasma-enhanced chemi-

\footnotetext{
a) Author to whom correspondence should be addressed; electronic mail: donglu.shi@uc.edu
}

cal vapor deposition. ${ }^{13-16}$ The plasma reactor for thin film deposition of nanotubes has been described in detail previously. ${ }^{8-12}$ The gases and monomers were introduced from the gas inlet during the plasma cleaning treatment and plasma polymerization. The system pressure was measured by a thermocouple pressure gauge. A rf power generator operating at $13.56 \mathrm{MHz}$ was used for the plasma film deposition. ${ }^{8,17-19}$

Before the plasma treatment, the chamber pressure was pumped down to less than $5 \mathrm{~Pa}$ at which time the monomer vapors were introduced into the reactor chamber. The operating pressure was adjusted by the mass flow controller. To be able to distinguish the deposited polymer thin film and the surface of carbon nanotubes, $\mathrm{C}_{6} \mathrm{~F}_{14}$ was used as the monomer for plasma polymerization. During plasma polymerization, the rf power used was $15 \mathrm{~W}$ and the system pressure was $30 \mathrm{~Pa}$. The plasma treatment time was $10 \mathrm{~min}$.

After the plasma treatment, the carbon nanotubes were examined using TEM, scanning electron microscopy (SEM), and TOF-SIMS. The HRTEM experiments were performed using a JEOL JEM 2010F electron microscope with a field emission source. The accelerating voltage was $200 \mathrm{kV}$. The SEM experiments were performed on a Philips XL30 FEG SEM. FTIR experiments were performed on a Perkin Elmer 1600 FTIR. TOF-SIMS was performed on a Ion-Tof model IV equipped with a $25 \mathrm{keV} 69 \mathrm{Ga} 1$ source. The mass resolution of the instrument was 8000 at 28 amu. Positive and negative spectra were collected in the mass range of $0-1000 \mathrm{amu}$. The spectra were acquired by rastering the beam over an area of $500 \times 500 \mu \mathrm{m}^{2}$ of bundle of the untreated or treated carbon nanotubes.

Figure 1(a) shows the SEM image of the original aligned carbon nanotubes. As can be seen in this figure, all the nanotubes grow in the same direction that is perpendicular to the 


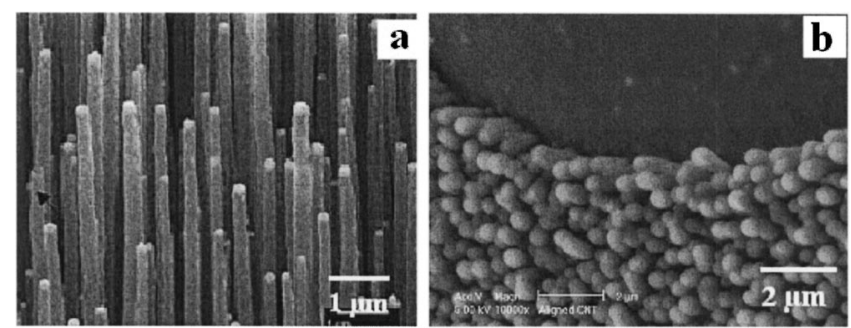

FIG. 1. SEM images of aligned carbon nanotubes (a) original; (b) plasma treated.

surface of the wafer. The diameters of nanotubes are around $200 \mathrm{~nm} .{ }^{14}$ An important surface morphology that needs to be pointed out is the rough surface of untreated nanotube. Figure 1(b) is the image of plasma treated aligned carbon nanotubes. Compared with Fig. 1(a), one can be see that the surface morphology exhibits smooth and round curvatures indicating coated polymer films. The diameters of coated nanotube are around $200-300 \mathrm{~nm}$, which is slightly greater than the uncoated nanotubes.

Figure 2(a) shows the cross-sectional bright-field TEM image of the original, uncoated aligned carbon nanotubes. The diameter of nanotube ranges from 50 to $200 \mathrm{~nm}$, consistent with the SEM observations (Fig. 1). All carbon nanotubes have a bamboo structure as shown in Fig. 2(b), which is similar to that of the CNTs grown from the Ni thin films prepared by sputtering using the same PECVD method. ${ }^{13-16}$ This HRTEM image [Fig. 2(b)] of a carbon nanotube also shows the graphitized wall of nanotubes. The TEM study shows that the surface of the nanotube exhibits an amorphous structure due to surface defects. This is a distinctively different surface feature observed from the random carbon nanotubes. Figure 2(c) is the bright-field TEM image of coated, aligned carbon nanotube showing a polymer thin film deposited on the outer surface of nanotube. The thickness of the film is approximately $20 \mathrm{~nm}$ and tightly bound to the nanotube. The film is identified as the typical amorphous structure by HRTEM observation [Fig. 2(d)]. For comparison, we show the interface image of a coated random carbon nanotube (PR-24-HZ), plasma treated under the same condi-

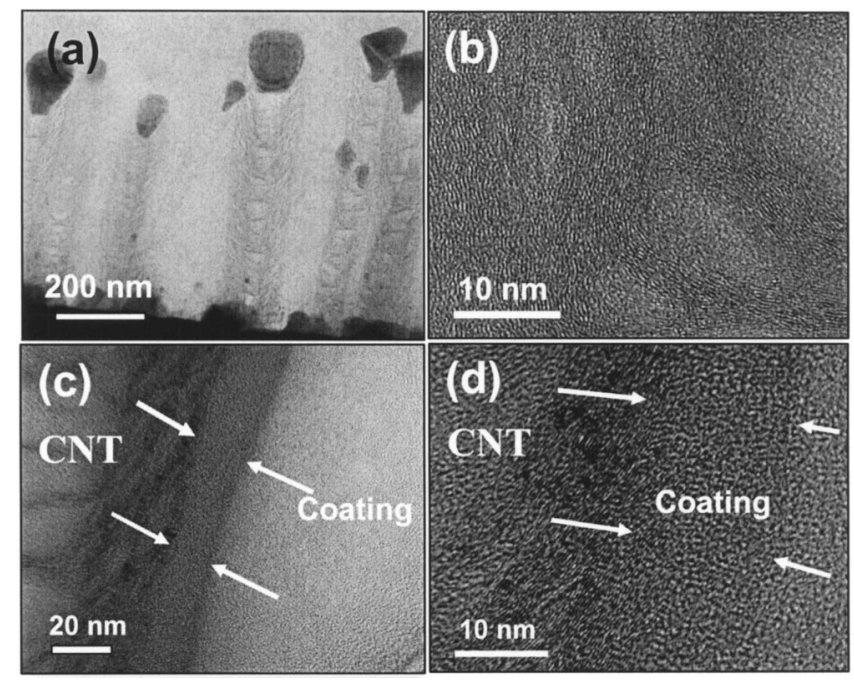

FIG. 2. (a) Bright-field TEM image of original aligned carbon nanotubes; (b) HRTEM image of the bamboo structure of the aligned carbon nanotube; (c) bright-field TEM image showing the plasma coated thin film on the nanotube surface; and (d) HRTEM image of the coating showing its amorphous structure and defected nanotube surface.
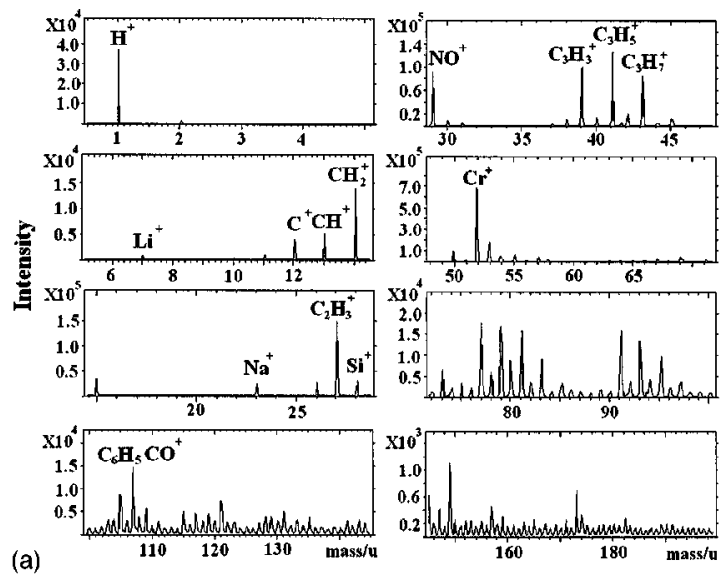

(a)

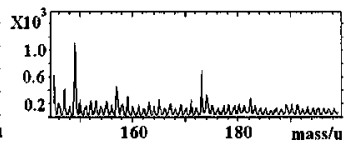

(b)
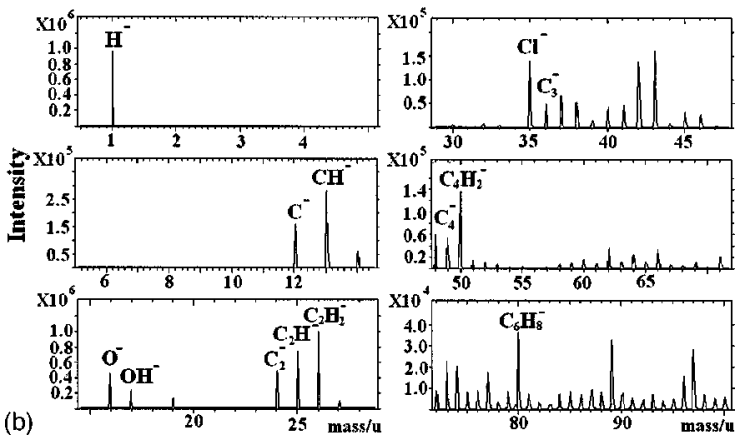

FIG. 3. SIMS spectra of original aligned carbon nanotubes, (a) positive ions and (b) negative ions.

tion from the previous experiments. ${ }^{11,12}$ A polymer thin film of $2 \mathrm{~nm}$ was observed on the carbon nanotube surface, and the carbon crystal lattices are well revealed.

To confirm the SEM and TEM observations (Figs. 1 and 2), TOF-SIMS was carried out to study the surface films of the aligned carbon nanotubes. Figure 3 shows a portion of the TOF-SIMS spectra of untreated aligned carbon nanotubes. The positive SIMS spectrum [Fig. 3(a)] of the untreated aligned carbon nanotubes show an appreciable intensity of chromium, which is from the buffer layer. There are also traces of $\mathrm{Na}, \mathrm{Si}, \mathrm{Mn}, \mathrm{Fe}, \mathrm{Ni}$, and Mo. Except for chromium, several strong hydrocarbon peaks, such as $\mathrm{C}^{+}, \mathrm{CH}^{+}$, $\mathrm{CH}_{2}^{+}, \mathrm{C}_{2} \mathrm{H}_{3}^{+}, \mathrm{C}_{3} \mathrm{H}_{3}^{+}, \mathrm{C}_{3} \mathrm{H}_{5}^{+}, \mathrm{C}_{3} \mathrm{H}_{7}^{+}$, and $\mathrm{C}_{6} \mathrm{H}_{5}-\mathrm{CO}^{+}$, appear in the positive spectra, which originate from the aligned carbon nanotubes. The negative SIMS spectra give more pronounced signal from the aligned carbon nanotubes. $\mathrm{C}^{-}, \mathrm{C}_{2}^{-}$, $\mathrm{C}_{3}^{-}, \mathrm{C}_{4}^{-}$, and $\mathrm{C}_{6} \mathrm{H}_{8}^{-}$, the typical species of nanotubes, can be easily identified from Fig. 3(b). Figures 4(a) and 4(b) are the SIMS spectra of the plasma treated aligned carbon nanotubes. Compared with Fig. 3(a), the strong chromium peaks are entirely suppressed as well as the hydrocarbon peaks (see Fig. 4). Instead, several carbonfluorine peaks, typically, $\mathrm{F}^{+}$, $\mathrm{CF}^{+}, \mathrm{CF}_{2}^{+}, \mathrm{C}_{2} \mathrm{~F}^{+}, \mathrm{CF}_{3}^{+}, \mathrm{C}_{3} \mathrm{~F}_{3}^{+}$, can be identified from Fig. 4(a). This spectrum indicates that the carbonfluorine thin film is not only uniformly deposited on the surface of the wafer substrate but also on the aligned carbon nanotubes. Figure 4(b) further confirms this characteristic by showing $\mathrm{F}^{-}, \mathrm{F}_{2}^{-}$, $\mathrm{CF}_{3}^{-}, \mathrm{COF}_{3}^{-}, \mathrm{C}_{3} \mathrm{~F}_{3}^{-}$peaks. These results are consistent with the HRTEM images shown in Fig. 2.

The chemical structure of the fluoropolymer films was investigated by FTIR spectroscopy. Since it was difficult to carry out FTIR directly on the coated aligned carbon nanotubes, the experiment was performed on the fluoropolymer films deposited on stainless steel substrate by using the same 

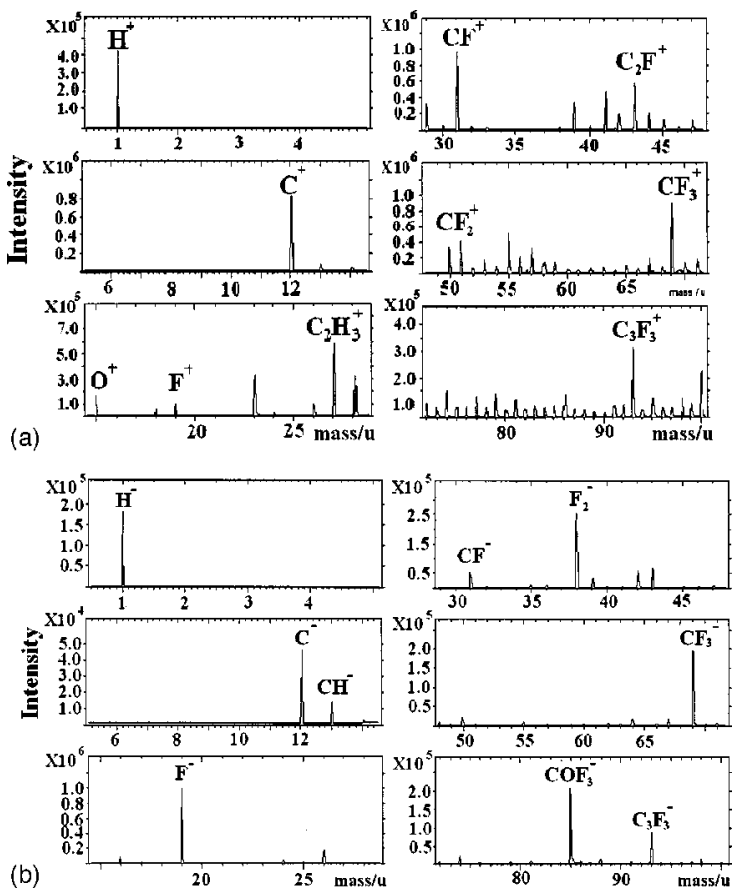

FIG. 4. SIMS spectra of plasma treated aligned carbon nanotubes, (a) positive ions and (b) negative ions.

condition for treating the aligned carbon nanotubes. As shown in Fig. 5, the FTIR spectrum of the fluoropolymer film exhibits the characteristic absorption bands of the $\mathrm{CF}_{2}$ groups at wave numbers of $517 \mathrm{~cm}^{-1}\left(\mathrm{CF}_{2}\right.$ wagging $)$, $643 \mathrm{~cm}^{-1}$ ( $\mathrm{CF}_{2}$ rocking), and $1155 \mathrm{~cm}^{-1}$ (asymmetric $\mathrm{C}-\mathrm{F}$ stretching). In particular, a strong absorption peak at $1518 \mathrm{~cm}^{-1}$ (aromatic C-F stretching) can be seen in Fig. 5. As is well known, there is no ring-like structure in $\mathrm{C}_{6} \mathrm{~F}_{14}$ monomer [Perfluorohexane, $\mathrm{CH}_{3}\left(\mathrm{CH}_{2}\right)_{4} \mathrm{CH}_{3}$ ]. Therefore, the ring-like structures observed in FTIR must be originated from plasma processing. In plasma polymerization processing, the coated film will form a highly cross-linked and ringlike structure which does not exist in the original monomer. Another remarkable difference between the monomer and coating after plasma processing is the existence of a new absorption peak at $1880 \mathrm{~cm}^{-1}$. This peak relates to the acid

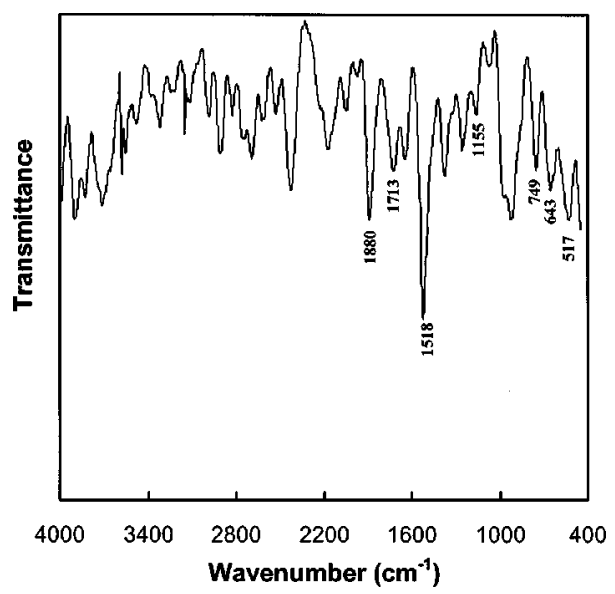

FIG. 5. FTIR spectrum of the $\mathrm{C}_{6} \mathrm{~F}_{14}$ on stainless steel substrate after plasma treatment. fluoride end group $-(\mathrm{C}=\mathrm{O})-\mathrm{F}$, which also does not exist in original monomer.

During coating, the monomer is introduced as a vapor and the collision frequency increases with the gas pressure. The rate of polymer condensation on the nanoparticle surfaces can be influenced by many parameters such as electron density, temperature, and energy density. To achieve a thin and uniform coating on such small nanoparticles, all these synthesis parameters must be optimized. Although a systematic study on the optimization of synthesis parameters has not yet been carried out, the preliminary experimental data have indicated that the polymer must be stable and not reactive with the substrate during coating. The gas pressure must be moderate for a low collision rate on the nanoparticle surfaces. In addition, polymerization should take place relatively fast after the condensation on the particle surfaces. These will ensure a uniform coating on the order of several nanometers for all particle sizes.

In summary, by using plasma polymerization, in this work, we have successfully deposited thin carbonfluorine films on aligned carbon nanotubes. The thin polymer film is uniformly coated on the surface of aligned carbon nanotubes and well characterized by TEM, SIMS, and FTIR.

This research is partially supported by a grant from the Ohio Aerospace Institute and by NSF NIRT 0304506. The work done at Boston College is supported by DOE under a grant DE-FG02-00ER45805.

${ }^{1}$ R. W. Siegel, Nanostruct. Mater. 3, 1 (1993).

${ }^{2}$ G. C. Hadjipanayis and R. W. Siegel, Nanophase Materials, SynthesisProperties-Applications (Kluwer, Dordrecht, 1994).

${ }^{3}$ G. M. Whitesides, J. P. Mathias, and C. T. Seto, Science 254, 1312 (1991).

${ }^{4}$ C. D. Stucky and J. E. MacDougall, Science 247, 669 (1990).

${ }^{5}$ H. Gleiter, Nanostruct. Mater. 6, 3 (1995).

${ }^{6} \mathrm{G}$. Timp, Nanotechnology (AIP, Melville, NY, 1998).

${ }^{7}$ Y. Bar-Cohen, 42nd AIAA Structures, Dynamics, and Materials Conference (SDM), Gossamer Spacecraft Forum (GSF), Seattle, WA, 2001, pp. $1-10$.

${ }^{8}$ D. Shi, S. X. Wang, W. J. van Ooij, L. M. Wang, J. Zhao, and Z. Yu, Appl. Phys. Lett. 78, 1234 (2001).

${ }^{9}$ D. Shi, P. He, J. Lian, L. M. Wang, and W. J. Vanooij, J. Mater. Res. 17, 2555 (2002).

${ }^{10}$ D. Shi, P. He, S. X. Wang, W. J. van Ooij, L. M. Wang, J. Zhao, and Z. Yu, J. Mater. Res. 17, 981 (2002).

${ }^{11}$ D. Shi, J. Lian, P. He, L. M. Wang, W. J. van Ooij, M. Schulz, Y. J. Liu, and D. B. Mast, Appl. Phys. Lett. 81, 5216 (2002).

${ }^{12}$ D. Shi, J. Lian, P. He, L. M. Wang, W. J. Van Ooij, M. Schulz, Y. J. Liu, and D. B. Mast, Appl. Phys. Lett. 83, 5301 (2003).

${ }^{13}$ Y. Tu, Z. P. Huang, D. Z. Wang, J. G. Wen, and Z. F. Ren, Appl. Phys. Lett. 80, 4018 (2002).

${ }^{14}$ Z. F. Ren, Z. P. Huang, J. W. Xu, J. H. Wang, P. Bush, M. P. Siegal, and P. N. Provencio, Science 282, 1105 (1998).

${ }^{15}$ Z. P. Huang, J. W. Xu, Z. F. Ren, J. H. Wang, M. P. Siegal, and P. N. Provencio, Appl. Phys. Lett. 73, 3845 (1998).

${ }^{16}$ J. H. Chen, Z. P. Huang, D. Z. Wang, S. X. Yang, J. G. Wen, and Z. F. Ren, Appl. Phys. A: Mater. Sci. Process. 73, 129 (2001).

${ }^{17}$ W. J. van Ooij, S. Luo, N. Zhang, and A. Chityala, in Proceedings International Conference on Advanced Manufacturing Technology (Science, New York, 1999), p. 1572.

${ }^{18}$ W. J. van Ooij and A. Chityala, Surface Modification of Powders by Plasma Polymerization, edited by K. L. Mittal (VSP, Utrecht, 2000), p. 243.

${ }^{19}$ W. J. van Ooij, N. Zhang, and S. Guo, in Fundamental and Applied Aspects of Chemically Modified Surfaces, edited by J. P. Blitz and C. B. Little (Royal Society of Chemistry, Cambridge, UK, 1999), p. 191. 